Jia-Bao Liu, Hafiz Muhammad Afzal Siddiqui, Muhammad Faisal Nadeem*, and Muhammad Ahsan Binyamin

\title{
Some topological properties of uniform subdivision of Sierpiński graphs
}

https://doi.org/10.1515/mgmc-2021-0006

received November 07, 2020; accepted January 01, 2021

Abstract: Sierpiński graphs are family of fractal nature graphs having applications in mathematics of Tower of Hanoi, topology, computer science, and many more diverse areas of science and technology. This family of graphs can be generated by taking certain number of copies of the same basic graph. A topological index is the number which shows some basic properties of the chemical structures. This article deals with degree based topological indices of uniform subdivision of the generalized Sierpiński graphs $S(n, G)$ and Sierpiński gasket $S_{n}$. The closed formulae for the computation of different kinds of Zagreb indices, multiple Zagreb indices, reduced Zagreb indices, augmented Zagreb indices, Narumi-Katayama index, forgotten index, and Zagreb polynomials have been presented for the family of graphs.

\section{Introduction}

Applications of molecular structure descriptors are a standard procedure in the study of structure-property relations nowadays, especially in the field of QSAR/QSPR study. During the last century, theoretical chemists started working on the use of topological indices to obtain

* Corresponding author: Muhammad Faisal Nadeem, Department of Mathematics, COMSATS University Islamabad, Lahore Campus, Pakistan, e-mail: mfaisalnadeem@ymail.com

Jia-Bao Liu: School of Mathematics and Physics, Anhui Jianzhu University, Hefei 230601, China; School of Mathematics, Southeast University, Nanjing 210096, China

Hafiz Muhammad Afzal Siddiqui: Department of Mathematics, COMSATS University Islamabad, Lahore Campus, Pakistan

Muhammad Ahsan Binyamin: Department of Mathematics, Government College University Faisalabad, Pakistan information on various properties of organic substances that depend upon their molecular structure. For this purpose, numerous topological indices were found and studied in the chemical literature (Todeschini and Cansonni, 2008). They used two zeroth order and two firstorder connectivity indices for the first time as descriptors in structure-property correlations in an optimization study. A set of new formulas for heat capacity, glass transition temperature, refractive index, cohesive energy, and dielectric constant were introduced - they were based on these descriptors. The Randić index has been used to parallel the boiling point and Kovats constants and was closely correlated with many chemical properties. A graph invariant that correlates the physico-chemical properties of a molecular graph with a number is called a topological index (Hansch and Leo, 1996). The first topological index was introduced by Wiener, a chemist, in 1947 to calculate the boiling points of paraffins in Wiener (1947). Zagreb indices, derived by Gutman and Trinajstić (1972), are used to study molecules and complexity of selected classes of molecules. Zagreb indices have found an interesting use in the QSPR/QSAR modeling and are useful in the study of anti-inflammatory activities of certain chemical instances.

A graph $S(n, G)$ is known as the generalized Sierpiński graph of $G$ of dimension $n$ having vertex set $\{1, \ldots, l\}^{n}$ and the set $\{t, s\}$ defines an edge iff $\exists i \in\{1, \ldots, n\}$ such as that:

- $t_{j}=s_{j}$ if $j<i$

- $t_{i} \neq s_{i}$ and $\left(t_{i}, s\right) \in E(G)$

- $t_{j}=s_{i}$ and $s_{j}=t_{i}$ if $j>i$.

Sierpiński graphs appear in many fields of mathematics and other branches of science (Beaudou et al., 2010). One of the most necessary sorts of Sierpiński graphs are the Sierpiński gasket graphs. The Sierpiński gasket graph is outlined as $S(n, k)$ with vertex set $(1,2,3 \ldots, n)$ and there is an edge between 2 vertices $x=\left(x_{1}, x_{2}, x_{3}, \ldots x_{n}\right)$ and there is an edge between 2 vertices $x=\left(x_{1}, x_{2}, x_{3}, \ldots x_{n}\right)$ and $y=\left(y_{1}, y_{2}, y_{3}, \ldots y_{n}\right)$ if and only if there is an $h \in[n]$ such that: 


$$
\begin{aligned}
& x_{j}=y_{j} \text { for } j=1,2,3 \ldots h-1 \\
& x_{h} \neq y_{h} \\
& x_{j}=y_{h} \text { and } y_{j}=x_{h} \text { for } j=h+1, \ldots n .
\end{aligned}
$$

The finite number of iterations in Sierpiński graphs gives Sierpiński gasket graphs and it is denoted by $S_{n}$. $S_{n}$ is consisted of three linked copies of $S_{n-1}$ that are up, down left, and downright elements of $S_{n}$. These graphs have been given by Scorer et al. (1944). These graphs play a vital role in dynamics system and probability and additionally as in psychological sciences. The generalized Sierpiński graph, $S(n, G)$, is constructed by repeating $|G|$ times $S(n-1, G)$ and adding one edge between replica $i$ and replica $j$ of $S(n-1, G)$ wherever $p q$ is an edge of $G$.

In this paper we will discuss the uniform subdivision of $S_{n}$ and $S(n, G)$. We will denote subdivision of $S_{n}$ by $S D\left(S_{n}\right)$ and subdivision of $S(n, G)$ by $S D(S(n, G))$. We study three types of Sierpiński graphs and now we will calculate the degree based topological indices for these types.

The first and second Zagreb indices which are also known as the graph invariants are the first vertex degree based structures descriptors defined by Gutman and Trinajstić (1972), and elaborated in Gutman et al. (1975) as $\sum_{p \in V(G)} d^{2}, \sum_{p q \in E(G)} d_{p} d_{q}$ and $\sum_{p \in V(G)} d^{3}$. These topological formula were first appeared in the in $\pi$ energy of conjugated molecules. After ten years, Balaban et al. (1983) included:

$$
M_{1}(G)=\sum_{p \in V(G)} d_{p}^{2}=\sum_{p q \in E(G)}\left(d_{p}+d_{q}\right)
$$

and $M_{2}(G)=\sum_{p q \in E(G)} d_{p} d_{q}$

Here $M_{1}(G)$ and $M_{2}(G)$ are known as the first and second Zagreb index. Gutman, Xu, and Das (Gutman and Das, 2004; Xu and Das, 2012) found the use Zagreb indices in QSPR and in QSAR. These topological indices have been used to study "molecular complexness", "chirality, ZE-isomorphism”, and Hetro-system. Their chemical applications and mathematical characteristics were studied by Das et al. (2013), Furtula et al. (2010), Gutman (2013, 2014), Nikolic et al. (2003), and Zhou (2004).

The term $\sum_{p \in V(G)}\left(d_{p}\right)^{3}$ remained forgotten for forty years. Recently, Furtula and Gutman (2015) have found the use of this term. They suggested that since this topological index was forgotten so it should be named as forgotten index or simply $F$ index it is defined as:

$$
F(G)=\sum_{p \in V(G)}\left(d_{p}\right)^{3}=\sum_{p q \in E(G)}\left[\left(d_{p}\right)^{2}+\left(d_{q}\right)^{2}\right] .
$$

Furtula and Gutman (2015) proved that the linear combination of $M_{1}+\lambda F$ has very accurate mathematical model of some physical properties of alkanes. Caporossi et al. (2010) introduced another index that is called a reduced second Zagreb index. Also this index has very important applications. It is defined as:

$$
R M_{2}(G)=\sum_{p q \in E(G)}\left(d_{p}-1\right)\left(d_{q}-1\right)
$$

Furtula et al. (2010) introduced the augmented Zagreb index in 2010. It is given as:

$$
A Z I(G)=\sum_{p q \in E(G)}\left(\frac{d_{q} d_{q}}{d_{q}+d_{q}-2}\right)^{3}
$$

Furtula et al. (2010) stated that it is a very important index for the study of the heat of formation in octanes and heptanes. If the exponent in $A Z I$ is replaces by -0.5 then it will become atom bound connectivity index. The results indicate that the AZI index gives the better results as compare to the $\mathrm{ABC}$ index.

Shirdel et al. (2013) gave the third Zagreb index, which is defined as:

$$
M_{3}(G)=\sum_{p q \in E(G)}\left(d_{p}+d_{q}\right)^{2}
$$

This index is combination of $F$ index and second Zagreb index, that is:

$$
M_{3}(G)=F(G)+2 M_{2}(G) \text {. }
$$

By using the above relation, we study the $F$ index with the Zagreb index.

Narumi and Katayama (1984) defined the degree product $P(G)=\prod_{p \in V(G)} d_{p}$. of a graph. It is defined as:

$$
N K(G)=\prod_{p \in V(G)} d_{p}
$$

Ghorbani and Azimi (2012) introduced the first and second multiple Zagreb indices, which are defines as:

$$
P M_{1}(G)=\prod_{p q \in V(G)}\left(d_{p}+d_{q}\right)=\prod_{p \in V(G)}\left(d_{p}\right)^{2}
$$


and $P M_{2}(G)=\prod_{p q \in V(G)}\left(d_{p} d_{q}\right)$

The first multiple Zagreb index is square of NarumiKatayama index.

Fathtabar (2009) defined the first and second Zagreb polynomial of the graph $G$ :

$$
Z G_{1}(G, y)=\sum_{p q \in E(G)} y^{d_{p}+d_{q}}
$$

and $Z G_{2}(G, y)=\sum_{p q \in E(G)} y^{d_{p} d_{q}}$

where $y$ is an attribute.

For more interesting articles and new developments in the area, please see: Akgunes et al. (2016), Bindusree et al. (2016), Das et al. (2013), Ji et al. (2020), Lokesha et al. (2018), Qu et al. (2019, 2020), and Togan et al. (2020).

In the following section some degree based topological indices for uniform subdivision of Sierpiński like graphs are studied.

\section{Zagreb indices and Zagreb polynomials for uniform subdivisions of Sierpiński gasket graphs $S D\left(S_{n}\right)$}

The graphs of uniform subdivision of Sierpiński gasket for $1 \leq n \leq 3$ is given in Figure 1 and the size of $S D\left(S_{n}\right)$ is $3^{n}$. There are 2 types of edges corresponding to the end vertices for $n>1$.

The partition of edge set of $S D\left(S_{n}\right)$ is given in Table 3.1.

$$
E_{d_{p,}, d_{q}}=\left(d_{p}, d_{q}\right)
$$

where $p q \in E(G)$.

The order of the $S D\left(S_{n}\right)$ is $\frac{1}{2}\left(3^{n}+3\right)+3^{n}$. In the vertex set there are 2 types of the vertices in $V(G)$ according to the degrees. In the Table 2 vertex partition of vertex set of $S D\left(S_{n}\right)$ is given below.

\section{Theorem 1}

The first and second Zagreb indices for $G=S D\left(S_{n}\right)$ is:

$$
M_{1}(G)=12\left(3^{n}-1\right) \text { and } M_{2}(G)=24\left(2 \times 3^{n-1}-1\right) \text {. }
$$
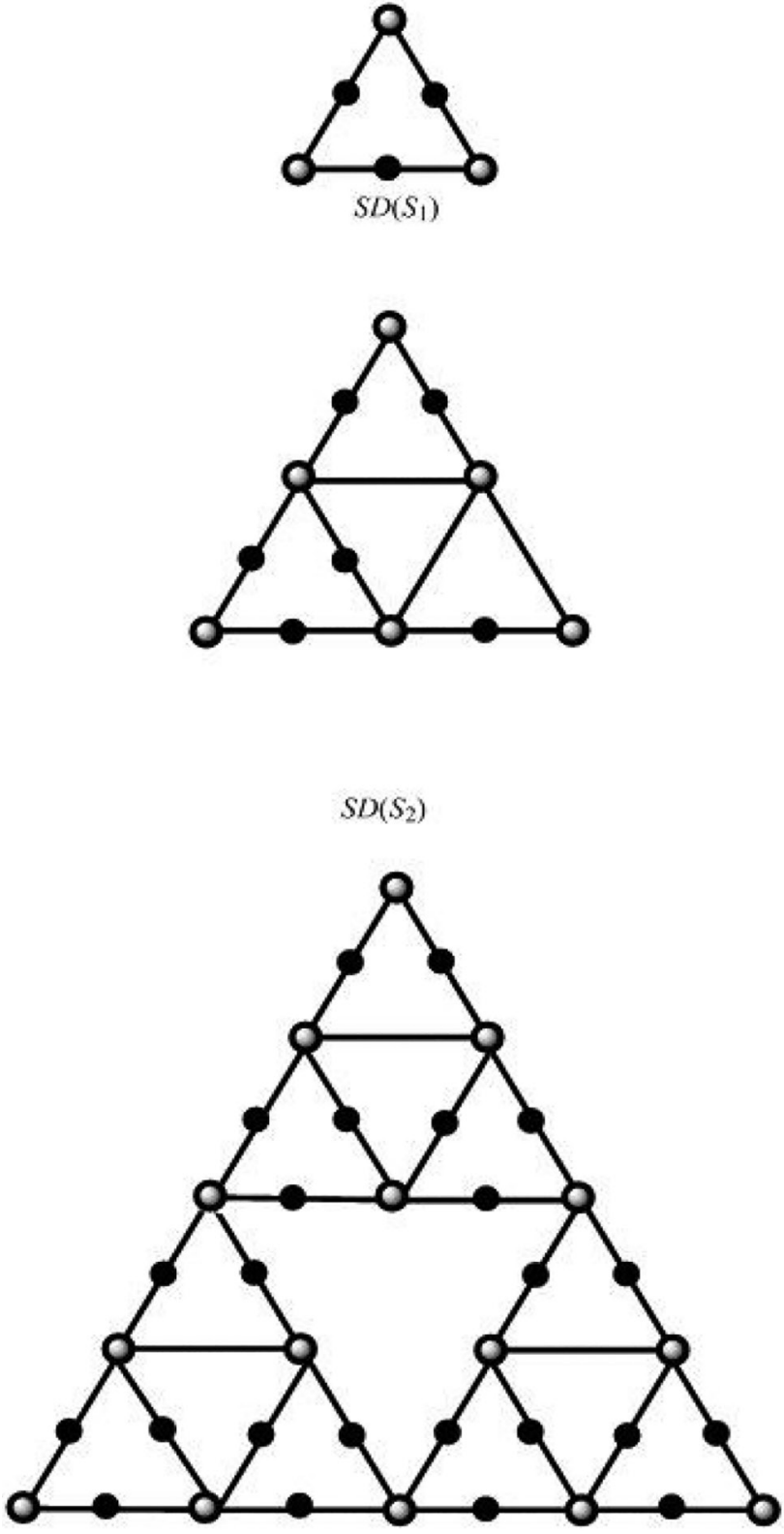

$S D\left(S_{3}\right)$

Figure 1: Uniform subdivision of Sierpiński gasket graph $S D\left(S_{1}\right)$, $S D\left(S_{2}\right)$, and $S D\left(S_{3}\right)$.

Table 1: The partition $E(G)$ of $S D\left(S_{n}\right)$

\begin{tabular}{lcc}
\hline$\left(\boldsymbol{d}_{p}, \boldsymbol{d}_{q}\right)$ & $(2,2)$ & $(2,4)$ \\
\hline Number of edges, $\left|E_{d_{p, d_{q}}}\right|$ & 6 & $2\left(3^{n}\right)-6$ \\
\hline
\end{tabular}

Table 2: Vertex partition of vertex set of $S D\left(S_{n}\right)$

\begin{tabular}{lcc}
\hline $\boldsymbol{d}_{p}$ & $\mathbf{2}$ & $\mathbf{4}$ \\
\hline Number of the vertices & $3^{n}+3$ & $\frac{1}{2}\left(3^{n}-3\right)$ \\
\hline
\end{tabular}


Proof: Using the equation $M_{1}(G)=\sum_{p q \in E(G)}\left(d_{p}+d_{q}\right)$ and the Table 1 we have the following result:

$$
\begin{aligned}
M_{1}(G) & =\sum_{p q \in E(G)}\left(d_{p}+d_{q}\right) \\
& =6 \times(2+2)+\left(2 \times 3^{n}-6\right) \times(2+4) \\
& =12\left(3^{n}-1\right) .
\end{aligned}
$$

Similarly, using the equation $M_{2}(G)=\sum_{p q \in E(G)} d_{p} d_{q}$ and Table 1 we have:

$$
\begin{aligned}
M_{2}(G) & =\sum_{p q \in E(G)} d_{p} \times d_{q} \\
& =6 \times(2 \times 2)+\left(2 \times 3^{n}-6\right) \times(2 \times 4) \\
& =24\left(2 \times 3^{n-1}-1\right) .
\end{aligned}
$$

\section{Theorem 2}

The reduced second Zagreb index for $\mathrm{G}=\mathrm{SD}\left(\mathrm{S}_{\mathrm{n}}\right)$ is:

$$
R M_{2}(G)=6\left(3^{n}-2\right) \text {. }
$$
Proof: Using the equation $R M_{2}(G)=\sum_{p q \in E(G)}\left(d_{p}-1\right)\left(d_{q}-1\right)$
and the Table 1 we have:

$$
\begin{aligned}
R M_{2}(G) & =\sum_{p q \in E(G)}\left(d_{p}-1\right)\left(d_{q}-1\right) \\
& =6 \times(2-1) \times(2-1)+\left(2 \times 3^{n}-6\right) \times(2-1) \times(4-1) \\
& =6\left(3^{n}-2\right) .
\end{aligned}
$$

\section{Theorem 3}

The third Zagreb index for $\mathrm{G}=\mathrm{SD}\left(\mathrm{S}_{\mathrm{n}}\right)$ is:

$$
M_{3}(G)=24\left(3^{n+1}-4\right) \text {. }
$$
Proof: Using the equation $M_{3}(G)=\sum_{p q \in E(G)}\left(d_{p}+d_{q}\right)^{2}$ and the
Table 1 we have:

$$
\begin{aligned}
M_{3}(G) & =\sum_{p q \in E(G)}\left(d_{p}+d_{q}\right)^{2} \\
& =6 \times(2+2)^{2}+\left(2 \times 3^{n}-6\right) \times(2+4)^{2} \\
& =24\left(3^{n+1}-4\right) .
\end{aligned}
$$

\section{Theorem 4}

The $F$ index for $\mathrm{G}=\mathrm{SD}\left(\mathrm{S}_{\mathrm{n}}\right)$ is:

$$
F(G)=32 \times 3^{n}-72 .
$$

Proof: By using the equation $F(G)=M_{3}(G)-2 M_{2}(G)$ and the Table 1 we have:

$$
\begin{aligned}
F(G) & =M_{3}(G)-2 M_{2}(G) \\
& =24\left(3^{n+1}-4\right)-2\left[8\left(2 \times 3^{n}-3\right)\right] \\
& \left.=8\left(5 \times 3^{n}-6\right)\right] .
\end{aligned}
$$

\section{Theorem 5}

The augmented Zagreb index for $\mathrm{G}=\mathrm{SD}\left(\mathrm{S}_{\mathrm{n}}\right)$ is:

$$
\operatorname{AZI}(G)=16 \times 3^{n} .
$$
Proof: Using the equation $A Z I(G)=\sum_{p q E E(G)}\left(\frac{d_{q} d_{q}}{d_{q}+d_{q}-2}\right)^{3}$
and the Table 1 we have:

$$
\begin{aligned}
A Z I(G) & =\sum_{p q \in E(G)}\left(\frac{d_{q} d_{q}}{d_{q}+d_{q}-2}\right)^{3} \\
& =6 \times\left(\frac{2 \times 2}{2+2-2}\right)^{3}+\left(2 \times 3^{n}-6\right) \times\left(\frac{2 \times 4}{2+4-2}\right)^{3} \\
& =16 \times 3^{n} .
\end{aligned}
$$

\section{Theorem 6}

The first and second multiple Zagreb indices for $\mathrm{G}=\mathrm{SD}\left(\mathrm{S}_{\mathrm{n}}\right)$ are:

$$
P M_{1}(G)=4^{6 n} \text { and } P M_{2}(G)=2^{6\left(3^{n}-1\right)} .
$$

Proof: Using the equation $P M_{1}(G)=\prod_{p \in V(G)}\left(d_{p}\right)^{2}$ and $P M_{2}(G)=\prod_{p q \in V(G)}\left(d_{p} d_{q}\right)$ and the Table 2 we have:

$$
\begin{aligned}
P M_{1}(G) & =\prod_{p \in V(G)}\left(d_{p}\right)^{2} \\
& =2^{2(3 n+3)} \times 4^{2 \times \frac{1}{2}\left(3^{n}-3\right)} \\
& =4^{6 n} .
\end{aligned}
$$

Here using the relation $P M_{2}(G)$ and the Table 1 we have:

$$
\begin{aligned}
P M_{2}(G) & =\prod_{p q \in V(G)}\left(d_{p} d_{q}\right) \\
& =(2 \times 2)^{6} \times(2 \times 4)^{\left(2 \times 3^{n}-6\right)} \\
& =2^{6 \times 3^{n}-6}=2^{6\left(3^{n}-1\right)} .
\end{aligned}
$$




\section{Theorem 7}

The Narumi-Katayama index for $\mathrm{G}=\mathrm{SD}\left(\mathrm{S}_{\mathrm{n}}\right)$ is:

$$
N K(G)=4^{3 n}
$$

Proof: Using the equation $N K(G)=\sqrt{P M_{1}(G)}$ we have:

$$
\begin{aligned}
N K(G) & =\sqrt{P M_{1}(G)} N K(G)=\sqrt{4^{6 n}} \\
& =4^{3 n} .
\end{aligned}
$$

\section{Theorem 8}

The first Zagreb polynomial for $\mathrm{G}=\mathrm{SD}\left(\mathrm{S}_{\mathrm{n}}\right)$ is:

$$
Z G_{1}(G, y)=6 y^{4}\left[1+y^{2}\left(3^{n-1}-1\right)\right] \text {. }
$$
Proof: Using the equation $Z G_{1}(G, y)=\sum_{p q E(G)} y^{d_{p}+d_{q}}$ and the
Table 1 we have:

$$
\begin{aligned}
Z G_{1}(G, y) & =6 \times y^{2+2}+\left(2 \times 3^{n}-6\right) \times y^{2+4} \\
& =6 y^{4}\left[1+y^{2}\left(3^{n-1}-1\right)\right] .
\end{aligned}
$$

\section{Theorem 9}

The first Zagreb polynomial for $\mathrm{G}=\mathrm{SD}\left(\mathrm{S}_{\mathrm{n}}\right)$ is:

$$
Z G_{2}(G, y)=6 y^{4}\left[1+y^{4}\left(3^{n-1}-1\right)\right]
$$
Proof: Using the equation $Z G_{2}(G, y)=\sum_{p q \in E(G)} y^{d_{p} d_{q}}$ and the
Table 1 we have:

$$
\begin{aligned}
Z G_{2}(G, y) & =\sum_{p q \in E(G)} y^{d_{p} d_{q}} \\
& =6 \times y^{2 \times 2}+\left(2 \times 3^{n}-6\right) \times y^{2 \times 4} \\
& =6 y^{4}\left[1+y^{4}\left(3^{n-1}-1\right)\right] .
\end{aligned}
$$

\section{Zagreb indices and Zagreb polynomials for uniform subdivisions of Sierpiński gasket graphs for $S D\left(S\left(n, C_{3}\right)\right)$}

The generalized Sierpiński graphs for the $S D\left(S\left(1, C_{3}\right)\right)$, $S D\left(S\left(2, C_{3}\right)\right)$, and $\operatorname{SD}\left(S\left(3, C_{3}\right)\right)$ are given in the Figure 2 . The size of $S D\left(S\left(n, C_{3}\right)\right)$ is $3\left(3^{n}-1\right)$. There are 2 types of edges corresponding to the degrees of end vertices for $n>1$. The edge partition for the set of $S D\left(S\left(n, C_{3}\right)\right)$ is given in the Table 3.

The order of the $S D\left(S\left(n, C_{3}\right)\right)$ is $\frac{3}{2}\left(3^{n}-1\right)+3^{n}$. In the vertex set there are 2 types of the vertices in $V(G)$ according to their degrees. The Table 4 illustrates a vertex partition of the $V(G)$ of $S D\left(S\left(n, C_{3}\right)\right)$.

The following theorems represents the formulas for Zagreb indices and Zagreb polynomials for $S D\left(S\left(n, C_{3}\right)\right)$.

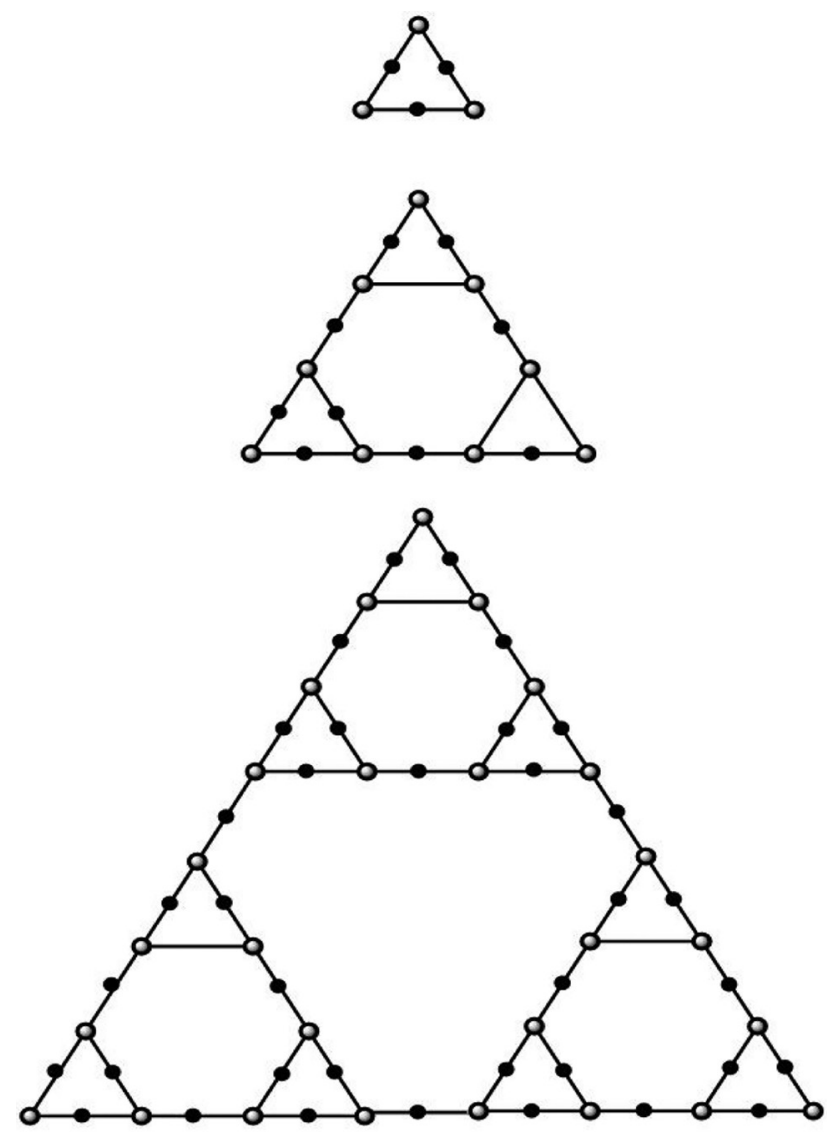

Figure 2: The generalized Sierpiński graphs for the $S D\left(S\left(1, C_{3}\right)\right)$, $S D\left(S\left(2, C_{3}\right)\right)$, and $S D\left(S\left(3, C_{3}\right)\right)$.

Table 3: The partition of $E(G)$ of $S D\left(S\left(n, C_{3}\right)\right)$

\begin{tabular}{lcc}
\hline$\left(\boldsymbol{d}_{p}, \boldsymbol{d}_{q}\right)$ & $(2,2)$ & $(2,3)$ \\
\hline Number of edges, $\left|E_{d_{p,}, d_{q}}\right|$ & 6 & $3^{n+1}-9$ \\
\hline
\end{tabular}

Table 4: Vertex partition of vertex set of $S D\left(S\left(n, C_{3}\right)\right)$

\begin{tabular}{lcc}
\hline $\boldsymbol{d}_{p}$ & $\mathbf{2}$ & $\mathbf{3}$ \\
\hline Number of the vertices & $\frac{3}{2}\left(3^{n}-1\right)+3$ & $3^{n}-3$ \\
\hline
\end{tabular}




\section{Theorem 10}

The first and second Zagreb indices for $\mathrm{SD}\left(\mathrm{S}\left(\mathrm{n}, \mathrm{C}_{3}\right)\right)$ are:

$$
M_{1}(G)=3\left(5 \times 3^{n}-7\right) \text { and } M_{2}(G)=6\left(3^{n+1}-5\right) \text {. }
$$

Proof: Using the equation $M_{1}(G)=\sum_{p q \in E(G)}\left(d_{p}+d_{q}\right)$ and the Table 3 we have the following results:

$$
\begin{aligned}
M_{1}(G) & =\sum_{p q \in E(G)}\left(d_{p}+d_{q}\right) \\
& =6 \times(2+2)+\left(3^{n+1}-9\right) \times(2+3) \\
& =3\left(5 \times 3^{n}-7\right) .
\end{aligned}
$$
Similarly, using the equation $M_{2}(G)=\sum_{p q \in E(G)} d_{p} d_{q}$ and
Table 3 we have:

$$
\begin{aligned}
M_{2}(G) & =\sum_{p q \in E(G)} d_{p} \times d_{q} \\
& =6 \times 2 \times 2+\left(3^{n+1}-9\right) \times(2 \times 3) \\
& =6\left(3^{n+1}-5\right) .
\end{aligned}
$$

\section{Theorem 11}

The reduced second Zagreb index for $\mathrm{SD}\left(\mathrm{S}\left(\mathrm{n}, \mathrm{C}_{3}\right)\right)$ is:

$$
R M_{2}(G)=6\left(3^{n}-2\right) \text {. }
$$

Proof: Using the equation $R M_{2}(G)=\sum_{p q \in E(G)}\left(d_{p}-1\right)\left(d_{q}-1\right)$
and the Table 3 we have:

$$
\begin{aligned}
R M_{2}(G) & =\sum_{p q \in E(G)}\left(d_{p}-1\right)\left(d_{q}-1\right) \\
& =6 \times(2-1) \times(2-1)+\left(3^{n+1}-9\right) \times(2-1) \times(3-1) \\
& =6\left(3^{n}-2\right) .
\end{aligned}
$$

\section{Theorem 12}

The third Zagreb index for $\mathrm{SD}\left(\mathrm{S}\left(\mathrm{n}, \mathrm{C}_{3}\right)\right)$ is:

$$
M_{3}(G)=25 \times 3^{n+1}-119 \text {. }
$$
Proof: Using the equation $M_{3}(G)=\sum_{p q \in E(G)}\left(d_{p}+d_{q}\right)^{2}$ and the
Table 3 we have:

$$
\begin{aligned}
M_{3}(G) & =\sum_{p q \in E(G)}\left(d_{p}+d_{q}\right)^{2} \\
& =6 \times(2+2)^{2}+\left(3^{n+1}-9\right) \times(2+3)^{2} \\
& =25 \times 3^{n+1}-119 .
\end{aligned}
$$

\section{Theorem 13}

The $F$ index for $\mathrm{SD}\left(\mathrm{S}\left(\mathrm{n}, \mathrm{C}_{3}\right)\right)$ is:

$$
F(G)=13 \times 3^{n+1}-59
$$

Proof: Using the equation $F(G)=M_{3}(G)-2 M_{2}(G)$ and the Theorem 11 and Theorem 12 we have:

$$
\begin{aligned}
F(G) & =M_{3}(G)-2 M_{2}(G) \\
& =25 \times 3^{n+1}-119-2\left[6\left(3^{n+1}-5\right)\right] \\
& =13 \times 3^{n+1}-59 .
\end{aligned}
$$

\section{Theorem 14}

The augmented Zagreb index for $\mathrm{SD}\left(\mathrm{S}\left(\mathrm{n}, \mathrm{C}_{3}\right)\right)$ is:

$$
A Z I(G)=24\left(3^{n}-1\right) .
$$

Proof: Using the equation $A Z I(G)=\sum_{p q \in E(G)}\left(\frac{d_{q} d_{q}}{d_{q}+d_{q}-2}\right)^{3}$ and
the Table 3 we have:

$$
\begin{aligned}
A Z I(G) & =\sum_{p q \in E(G)}\left(\frac{d_{q} d_{q}}{d_{q}+d_{q}-2}\right)^{3} \\
& =6 \times\left(\frac{2 \times 2}{2+2-2}\right)^{3}+\left(3^{n+1}-9\right) \times\left(\frac{2 \times 3}{2+3-2}\right)^{3} \\
& =24\left(3^{n}-1\right) .
\end{aligned}
$$

\section{Theorem 15}

The first and second multiple Zagreb indices for $\mathrm{SD}\left(\mathrm{S}\left(\mathrm{n}, \mathrm{C}_{3}\right)\right)$ are:

$$
P M_{1}(G)=2^{3\left(3^{n}+1\right)} \times 3^{6\left(3^{n-1}-1\right)} \text { and } P M_{2}(G)=2^{3\left(3^{n}+1\right)} \times 3^{\left(3^{n+1}-9\right)} \text {. }
$$

Proof: Using the equation $P M_{1}(G)=\prod_{p \in V(G)}\left(d_{p}\right)^{2}$ and $P M_{2}(G)=\prod_{p q \in V(G)}\left(d_{p} d_{q}\right)$ and the Table 4 we have: 


$$
\begin{aligned}
P M_{1}(G) & =\prod_{p \in V(G)}\left(d_{p}\right)^{2} \\
& =4^{\frac{3}{2}\left(3^{n}-1\right)+3} \times 9^{\left(3^{n}-3\right)} \\
& =2^{3\left(3^{n}+1\right)} \times 3^{6\left(3^{n-1}-1\right)} .
\end{aligned}
$$

Here using the relation $P M_{2}(G)$ and the Table 3 we have:

$$
\begin{aligned}
P M_{2}(G) & =\prod_{p q \in V(G)}\left(d_{p} d_{q}\right) \\
& =4^{6} \times 6^{\left(3^{n+1}-9\right)} \\
& =2^{3\left(3^{n}+1\right)} \times 3^{\left(3^{n+1}-9\right)} .
\end{aligned}
$$

\section{Theorem 16}

The Narumi-Katayama index for $\mathrm{SD}\left(\mathrm{S}\left(\mathrm{n}, \mathrm{C}_{3}\right)\right)$ is:

$$
N K(G)=2^{\frac{3}{2}\left(3^{n}+1\right)} \times 3^{\left(3^{n}-3\right)} .
$$

Proof: Using the equation $N K(G)=\sqrt{P M_{1}(G)}$ we have:

$$
\begin{aligned}
N K(G) & =\sqrt{P M_{1}(G)} \\
& =\sqrt{2^{3\left(3^{n}+1\right)} \times 3^{2\left(3^{n}-3\right)}} \\
& =2^{2^{3}\left(^{n}+1\right)} \times 3^{\left(3^{n}-3\right)} .
\end{aligned}
$$

\section{Theorem 17}

The first Zagreb polynomial for $\mathrm{SD}\left(\mathrm{S}\left(\mathrm{n}, \mathrm{C}_{3}\right)\right)$ is:

$$
Z G_{1}(G, y)=3 y^{4}\left[2-3 y\left(1-2 \times 3^{n-1}\right)\right] \text {. }
$$
Proof: Using the equation $Z G_{1}(G, y)=\sum_{p q \in E(G)} y^{d_{p}+d_{q}}$ and the
Table 3 we have:

$$
\begin{aligned}
& =6 \times y^{2+2}+\left(3^{n+1}-9\right) \times y^{2+3} \\
& =3 y^{4}\left[2-3 y\left(1-2 \times 3^{n-1}\right)\right] .
\end{aligned}
$$

\section{Theorem 18}

The first Zagreb polynomial for $\mathrm{SD}\left(\mathrm{S}\left(\mathrm{n}, \mathrm{C}_{3}\right)\right)$ is:

$$
Z G_{2}(G, y)=3 y^{4}\left[2-3 y^{2}\left(1-3^{n-1}\right)\right] .
$$
Proof: Using the equation $Z G_{2}(G, y)=\sum_{p q \in E(G)} y^{d_{p} d_{q}}$ and the
Table 3.3 we have:

$$
\begin{aligned}
Z G(G y) & \sum_{p q \in E(G)} y^{d_{p} d_{q}} \\
= & 6 \times y^{2 \times 2}+\left(3^{n+1}-9\right) \times y^{2 \times 3} \\
= & 3 y^{4}\left[2-3 \mathrm{y}^{2}\left(1-3^{n-1}\right)\right] .
\end{aligned}
$$

\section{Zagreb indices and Zagreb polynomials for uniform subdivisions of Sierpiński gasket graphs for $S D\left(S\left(n, C_{4}\right)\right)$}

The generalized Sierpiński graphs for the $S D\left(S\left(1, C_{4}\right)\right)$, $S D\left(S\left(2, C_{4}\right)\right)$, and $\operatorname{SD}\left(S\left(3, C_{4}\right)\right)$ are given in the Figure 3. The size of $S D\left(S\left(n, C_{4}\right)\right)$ is $\frac{8}{3}\left(4^{n}-1\right)$. There are 2 types of edges corresponding to the degrees of end vertices for $n>1$. The partition for the edges of the set of $S D\left(S\left(n, C_{4}\right)\right)$ is shown in the Table 5.

The order of the $S\left(n, C_{4}\right)$ is $\frac{4}{3}\left(4^{n}-1\right)+4^{n}$. In the vertex set there are 2 types of the vertices in $\mathrm{V}(\mathrm{G})$ according to the degrees. The Table 4 represents vertex partition of $V(G)$ of $S D\left(S\left(n, C_{4}\right)\right)$.

The following theorems represent the formulas for Zagreb indices and Zagreb polynomials for $S D\left(S\left(n, C_{4}\right)\right)$.

\section{Theorem 19}

The first and second Zagreb indices for $\mathrm{SD}\left(\mathrm{S}\left(\mathrm{n}, \mathrm{C}_{4}\right)\right)$ are:

$$
M_{1}(G)=\frac{4}{3}\left(38 \times 4^{n-1}+1\right) \text { and } M_{2}(G)=\frac{2}{3}\left(22 \times 4^{n}-35\right) \text {. }
$$

Proof: Using the equation $M_{1}(G)=\sum_{p q \in E(G)}\left(d_{p}+d_{q}\right)$ and the Table 5 we have the following results:

$$
\begin{aligned}
M_{1}(G) & =\sum_{p q \in E(G)}\left(d_{p}+d_{q}\right) \\
& =\frac{2}{3}\left(4^{n}+8\right) \times(2+2)+2\left(4^{n}-4\right) \times(2+3) \\
& =\frac{4}{3}\left(38 \times 4^{n-1}+1\right) .
\end{aligned}
$$






Figure 3: The generalized Sierpiński graphs for the $S D\left(S\left(1, C_{4}\right)\right)$, $S D\left(S\left(2, C_{4}\right)\right)$, and $S D\left(S\left(3, C_{4}\right)\right)$.

Table 5: The partition $\mathrm{E}(\mathrm{G})$ of $S D\left(S\left(n, C_{4}\right)\right)$

\begin{tabular}{lcc}
\hline$\left(\boldsymbol{d}_{p}, \boldsymbol{d}_{q}\right)$ & $(2,2)$ & $(2,3)$ \\
\hline Number of edges, $\left|E_{d_{p}, d_{q}}\right|$ & $\frac{2}{3}\left(4^{n}+8\right)$ & $2\left(4^{n}-4\right)$ \\
\hline
\end{tabular}

Similarly using the equation $M_{2}(G)=\sum_{p q \in E(G)} d_{p} d_{q}$ and

$$
\begin{aligned}
M_{2}(G) & =\sum_{p q \in E(G)} d_{p} \times d_{q} \\
& =\frac{2}{3}\left(4^{n}+8\right) \times(2 \times 2)+2\left(4^{n}-4\right) \times(2 \times 3) \\
& =\frac{2}{3}\left(22 \times 4^{n}-35\right) .
\end{aligned}
$$

Theorem 20:

The reduced second Zagreb index for $\mathrm{SD}\left(\mathrm{S}\left(\mathrm{n}, \mathrm{C}_{4}\right)\right)$ is:

$$
R M_{2}(G)==\frac{2}{3}\left(7 \times 4^{n}-16\right)
$$

Proof: Using the equation $R M_{2}(G)=\sum_{p q \in E(G)}\left(d_{p}-1\right)\left(d_{q}-1\right)$
and the Table 5 we have:

$$
\begin{aligned}
R M_{2}(G) & =\sum_{p q \in E(G)}\left(d_{p}-1\right)\left(d_{q}-1\right) \\
& =\frac{2}{3}\left(4^{n}+8\right) \times(1 \times 1)+2\left(4^{n}-4\right) \times(1 \times 2) \\
& =\frac{2}{3}\left(7 \times 4^{n}-16\right) .
\end{aligned}
$$

\section{Theorem 21}

The third Zagreb index for $\mathrm{SD}\left(\mathrm{S}\left(\mathrm{n}, \mathrm{C}_{4}\right)\right)$ is:

$$
M_{3}(G)=\frac{2}{3}\left(91 \times 4^{n}-172\right) \text {. }
$$
Proof: Using the equation $M_{3}(G)=\sum_{p q \in E(G)}\left(d_{p}+d_{q}\right)^{2}$ and the
Table 5 we have:

$$
\begin{aligned}
M_{3}(G) & =\sum_{p q \in E(G)}\left(d_{p}+d_{q}\right)^{2} \\
& =\frac{2}{3}\left(4^{n}+8\right) \times\left(4^{2}\right)+2\left(4^{n}-4\right) \times 5^{2} \\
& =\frac{2}{3}\left(91 \times 4^{n}-172\right) .
\end{aligned}
$$

\section{Theorem 22}

The $F$ index for $\mathrm{SD}\left(\mathrm{S}\left(\mathrm{n}_{4} \mathrm{C}_{4}\right)\right)$ is:

$$
F(G)=\frac{2}{3}\left(47 \times 4^{n}-102\right)
$$

Proof: Using the equation $F(G)=M_{3}(G)-2 M_{2}(G)$ and the Theorem 20 and Theorem 21 we have:

$$
\begin{aligned}
F(G) & =M_{3}(G)-2 M_{2}(G) \\
& =\frac{2}{3}\left(91 \times 4^{n}-172\right)-\frac{2 \times 2}{3}\left(22 \times 4^{n}-35\right) \\
& =\frac{2}{3}\left(47 \times 4^{n}-102\right) .
\end{aligned}
$$

\section{Theorem 23}

The augmented Zagreb index for $\mathrm{SD}\left(\mathrm{S}\left(\mathrm{n}, \mathrm{C}_{4}\right)\right)$ is:

$$
A Z I(G)=\frac{64}{3}\left(4^{n}-1\right) \text {. }
$$


Proof: Using the equation $A Z I(G)=\sum_{p q \in E(G)}\left(\frac{d_{q} d_{q}}{d_{q}+d_{q}-2}\right)^{3}$
and the Table 5 we have:

$$
\begin{aligned}
A Z I(G) & =\frac{2}{3}\left(4^{n}+8\right) \times(2)^{3}+2\left(4^{n}-4\right) \times(2)^{3} \\
& =\frac{64}{3}\left(4^{n}-1\right) .
\end{aligned}
$$

\section{Theorem 24}

The first and second multiple Zagreb indices for $\mathrm{SD}\left(\mathrm{S}\left(\mathrm{n}, \mathrm{C}_{4}\right)\right)$ are:

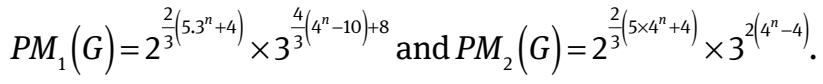

Proof: Using the equation $P M_{1}(G)=\prod_{p \in V(G)}\left(d_{p}\right)^{2}$ and $P M_{2}(G)=\prod_{p q \in V(G)}\left(d_{p} d_{q}\right)$ and the Table 6 we have:

$$
\begin{aligned}
P M_{1}(G) & =\prod_{p \in V(G)}\left(d_{p}\right)^{2} \\
& =2^{\frac{2}{3}\left(5 \cdot 3^{n}+4\right)} \times 3^{\frac{4}{3}\left(4^{n}-10\right)+8} .
\end{aligned}
$$

Here using the relation $P M_{2}(G)$ and the Table 5 we have:

$$
\begin{aligned}
P M_{2}(G) & =\prod_{p q \in V(G)}\left(d_{p} d_{q}\right) \\
& =4^{\frac{2}{3}\left(4^{n}+8\right)} \times 6^{2\left(4^{n}-4\right)} \\
& =2^{\frac{2}{3}\left(5 \times 4^{n}+4\right)} \times 3^{2\left(4^{n}-4\right)} .
\end{aligned}
$$

\section{Theorem 25}

The Narumi-Katayama index for $\operatorname{SD}\left(S\left(n, C_{4}\right)\right)$ is:

$$
N K(G)=2^{\frac{1}{3}\left(5.3^{n}+4\right)} \times 3^{\frac{2}{3}\left(4^{n}-10\right)+4} .
$$

Table 6: Vertex partition of vertex set of $S D\left(S\left(n, C_{4}\right)\right)$

\begin{tabular}{lcc}
\hline $\boldsymbol{d}_{p}$ & 2 & 3 \\
\hline Number of the vertices & $\frac{1}{3}\left(5.4^{n}+4\right)$ & $\frac{2}{3}\left(4^{n}-10\right)+4$ \\
\hline
\end{tabular}

Proof: Using the equation $N K(G)=\sqrt{P M_{1}(G)}$ we have:

$$
\begin{aligned}
& =\sqrt{2^{\frac{2}{3}\left(5 \cdot 3^{n}+4\right)} \times 3^{\frac{4}{3}\left(4^{n}-10\right)+8}} \\
& =2^{\frac{1}{3^{\left(5.3^{n}+4\right)}} \times 3^{\frac{2}{3}\left(4^{n}-10\right)+4}} .
\end{aligned}
$$

\section{Theorem 26}

The first Zagreb polynomial for $\mathrm{SD}\left(\mathrm{S}\left(\mathrm{n}, \mathrm{C}_{4}\right)\right)$ is:

$$
Z G_{1}(G, y)=8 y^{4}\left[\frac{1}{3}\left(4^{n-1}+2\right)+\left(4^{n-1}-1\right) \times y\right] \text {. }
$$
Proof: Using the equation $Z G_{1}(G, y)=\sum_{p q \in E(G)} y^{d_{p}+d_{q}}$ and the
Table 5 we have:

$$
\begin{aligned}
& =\frac{2}{3}\left(4^{n}+8\right) \times y^{4}+2\left(4^{n}-4\right) \times y^{5} \\
& =8 y^{4}\left[\frac{1}{3}\left(4^{n-1}+2\right)+\left(4^{n-1}-1\right) \times y\right] .
\end{aligned}
$$

\section{Theorem 27}

The first Zagreb polynomial for $\operatorname{SD}\left(\mathrm{S}\left(\mathrm{n}, \mathrm{C}_{4}\right)\right)$ is:

$$
Z G_{2}(G, y)=8 y^{4}\left[\frac{1}{3}\left(4^{n-1}+2\right)+\left(4^{n-1}-1\right) \times y^{2}\right]
$$
Proof: Using the equation $Z G_{2}(G, y)=\sum_{p q \in E(G)} y^{d_{p} d_{q}}$ and the
Table 5 we have:

$$
\begin{aligned}
Z G_{2}(G, y) & =\sum_{p q \in E(G)} y^{d_{p} d_{q}} \\
& =\frac{2}{3}\left(4^{n}+8\right) \times y^{4}+2\left(4^{n}-4\right) \times y^{6} \\
& =8 y^{4}\left[\frac{1}{3}\left(4^{n-1}+2\right)+\left(4^{n-1}-1\right) \times y^{2}\right] .
\end{aligned}
$$

\section{Conclusion}

Topological indices are often studied with the help of their descriptors. In this paper certain degree based topological indices namely Zagreb indices, multiple Zagreb indices, reduced Zagreb indices, augmented Zagreb indices, Narumi-Katayama index, forgotten 
index, and Zagreb polynomials have been studied by subdivision of three families of Sierpiński graphs namely $S D\left(S_{n}\right), S D\left(S\left(n, C_{3}\right)\right)$ and $S D\left(S\left(n, C_{4}\right)\right)$. In future we will pay attention by subdividing the other families of Sierpiński graphs.

Acknowledgement: The authors are grateful to anonymous referees and editor for their valuable suggestions/criticism which helped us to improve the quality of the paper.

Funding information: This work is supported in part by the China Postdoctoral Science Foundation under Grant 2017M621579, in part by the Postdoctoral Science Foundation of Jiangsu Province under Grant 1701081B, in part by the Project of Anhui Jianzhu University under Grant 2016QD116. Natural Science Fund of Education Department of Anhui Province under Grant KJ2020A0478.

Author contributions: Jia-Bau Liu: Project administration, Writing - original draft; Hafiz Muhammad Afzal Siddiqui: Methodology visualization; Muhammad Faisal Nadeem: Formal analysis, Visualization; Muhammad Ahsan Binyamin: Formal analysis, Writing - review and editing.

Conflict of interest: Authors state no conflict of interest.

Data availability statement: All data are provided in the manuscript.

\section{References}

Akgunes N., Das K.C., Togan M., Yurttas A., Cangul I.N., Cevik A.S., On the first Zagreb index and multiplicative Zagreb coindices of graphs. An. Sti. U. Ovid. Co-Mat. 2016, 24(1), 153-176.

Balaban A.T., Motoc I., Bonchev D., Makenyan O., Topological indices forstructure-activity correlations. Top. Curr. Chem., 1983, 114, 21-55.

Beaudou L., Gravier S., Klavžar S., Kovse M., Mollard M., Covering codes in Sierpiński graphs. Discrete. Math. Theor., 2010, $12,63-74$.

Bindusree A.R., Cangul I.N., Lokesha V., Cevik A.S., Zagreb polynomials of three graph operators. Filomat, 2016, 30(7), 1979-1986.

Caporossi G., Hansen P. Vukičević D., Comparing of Zagreb indices of cylic graphs. MATCH Commun. Math. Comput. Chem., 2010, 63, 441-451.

Das K.C., Togan M., Yurttas A., Cevik A.S., Cangul I.N., The multiplicative Zagreb indices of graph operations. J. Inequal. Appl., 2013, 90, 2013.
Fathtabar H., Zagreb polynomials and pi indices of some nanostructures. Dig. J. Nanomater. Bios., 2009, 4, 189-191.

Furtula B., Graovac A., Vukičević D. Augmented Zagreb index. J. Math. Chem., 2010, 48, 370-380.

Furtula B., Gutman I., A forgotten topological index. J. Math. Chem., 2015, 53, 1184-1190.

Ghorbani M., Azimi N., Note on multiple Zagreb indices. Iran. J. Math. Chem., 2012, 3, 137-143.

Gutman I., Degree-based topological indices. Croat. Chem. Acta, 2013, 86, 351-361.

Gutman I., An exceptional property of first Zagreb index. MATCH-Commun. Math. Co., 2014, 72, 733-740.

Gutman I., Das K.C., The first Zagreb index 30 years after. MATCH-Commun. Math. Co., 2004, 50, 83-92.

Gutman I., Trinajstić N., Total $\varphi$-electron energy of alternant hydrocarbons. Chem. Phys. Lett., 1972, 17, 535-538.

Gutman I., Ruščić B., Trinajstić N., Wilcox C.F., Graph theory and molecular orbitals. XII - Acyclic Polyenes. J. Chem. Phys., 1975, 62, $1692-1704$.

Hansch C., Leo L., Exploring QSAR fundamentals and applicability in chemistry and biology. J. Am. Chem. Soc., 1996.

Ji Y., Qu S., Wu Z., Liu Z., A fuzzy-robust weighted approach for multicriteria bilevel games. IEEE T. Ind. Inform., 2020, 16(8), 5369-5376.

Lokesha V., Shruti R., Ranjini P.S., Cevik A.S., On certain topological indices of nanostructures using $Q(G)$ and $R(G)$ operators. Commun. Fac. Sci. Univ. Ank. Ser. A1 Math. Stat., 2018, 66(2), 178-187.

Narumi H., Katayama H., Simple topological index a newly devised index characterizing the topological nature of structural isomers of saturated hydrocarbons. Mem. Fac. Eng. Hokkaido Univ., 1984, 16, 209-214.

Nikolić S., Kovačević G., Miličević A., Trinajstić N., The Zagreb indices 30 years after. Croat. Chem. Acta, 2003, 76, 113-124.

Qu S., Han Y., Wu Z., Raza H., Consensus modeling with as asymmetric cost based on data-driven robust optimization. Group Decis. Negot., 2020, DOI: 10.1007/s10726-020-09707-w

Qu S., Zhou Y., Zhang Y., Wahab M.I.M., Zhang G., Ye Y., Optimal strategy for a green supply chain considering shipping policy and default risk. Comput. Ind. Eng, 2019, 131, 172-186.

Scorer R.S., Grundy P.M., Smith C.A.B., Some binary games. Math. Gaz., 1944, 28(280), 96-103.

Shirdel G.H., Rezapour H. Sayadi A.M., The hyper-Zagreb index of graph operations. Iran. J. Math. Chem., 2013, 4, 213-220.

Todeschini R., Consonni V., Handbook of molecular descriptors, methods and principles in medicinal chemistry. Wiley, New York, 2008.

Togan M., Yurttas A., Cevik A.S., Cangul I.N., Zagreb indices and multiplicative Zagreb indices of double graphs of subdivision graphs. TWMS J. App. Eng. Math. 2019, 9(2), 404-412.

Wiener H., Structural determination of paraffin boiling point. J. Am. Chem. Soc., 1947, 69, 17-20.

Xu K., Das K.C., Zagreb indices and polynomials of TUHRC4 and TUSC4C8 nanotubes. MATCH-Commun. Math. Co., 2012, 68, 257-272.

Zhou B., Zagreb indices. MATCH-Commun. Math. Co., 2004, 52, 113-118. 